\title{
Periodic forcing in viscous fingering of a nematic liquid crystal
}

\author{
R. Folch, ${ }^{1,2, *}$ T. Tóth-Katona, ${ }^{3, \dagger}$ Á. Buka, ${ }^{3}$ J. Casademunt, ${ }^{1}$ and A. Hernández-Machado ${ }^{1,2}$ \\ ${ }^{1}$ Departament d'Estructura i Constituents de la Matèria, Universitat de Barcelona, Avinguda Diagonal, 647, E-08028-Barcelona, Spain \\ ${ }^{2}$ Groupe de Physique des Solides, UMR 7588, CNRS / Universités Paris VII et Paris VI, Tour 23, 2 place Jussieu, \\ F-75251 Paris Cedex 05, France \\ ${ }^{3}$ Research Institute for Solid State Physics and Optics, Hungarian Academy of Sciences, P. O. Box 49, H-1525 Budapest, Hungary
}

(Received 9 June 2000; published 25 October 2001)

\begin{abstract}
Viscous fingering of an air-nematic interface in a radial Hele-Shaw cell is studied when periodically switching on and off an electric field, which reorients the nematic and thus changes its viscosity, as well as the surface tension and its anisotropy (mainly enforced by a single groove in the cell). Undulations at the sides of the fingers are observed that correlate with the switching frequency and with tip oscillations that give maximal velocity to smallest curvatures. These lateral undulations appear to be decoupled from spontaneous (noise induced) side branching. It is concluded that the lateral undulations are generated by successive relaxations between two limiting finger widths. The change between these two selected pattern scales is mainly due to the change in the anisotropy. This scenario is confirmed by numerical simulations in the channel geometry, using a phase-field model for anisotropic viscous fingering.
\end{abstract}

DOI: 10.1103/PhysRevE.64.056225 PACS number(s): 47.54.+r, 61.30.-v, 47.20.Ma, 47.20.Hw

\section{INTRODUCTION}

Interfacial instabilities constitute a diverse domain in nonequilibrium pattern formation, with examples ranging from biology (e.g., bacterial growth) to mathematics (Stefan problems), passing by physical systems as flows in porous media, solidification, electrodeposition or flame propagation [1].

Progress in this domain has usually been made by studying prototype systems as solidification or viscous fingering. The latter deals with the destabilization of the interface between two immiscible fluids when the more viscous fluid is displaced by the less viscous one, which is either injected at an end of a channel-shaped cell (channel geometry) or from the center of the cell (radial geometry) - for a review see Ref. [2]. This initial destabilization leads to the formation of fingers in both geometries, which finally restabilize into a single stationary finger in the channel one. However, a sufficient amount of noise may cause this single finger to tip split. In contrast, in the isotropic, radial cell, fingers do not stabilize, but repeatedly tip split to form more and more fingers [3].

An external perturbation, however, can dramatically change the fingers and can even suppress the tip splitting in both geometries. A bubble of gas trapped just in front of an advancing finger causes tip stabilization and (eventually) intensive and very regular side branching both in the radial [4] and channel [5] geometries. Engraving a grid on one of the plates of the radial cell introduces an anisotropy, which, if strong enough, also inhibits tip splitting and produces dendrites and faceted structures, resulting in a rich morphology diagram [6,7]. Different etched lattices give a variety of highly branched structures whose symmetry depends on that of the lattice when the anisotropy it introduces is strong

\footnotetext{
*Email addresses: roger@gps.jussieu.fr and roger@ecm.ub.es

†Present address: Physics Department, Kent State University, P.O. Box 5190, Kent, OH 44242
}

enough [8]. The replacement of the grid by a set of parallel grooves [9] has produced an even more complicated morphology diagram than that presented in Ref. [6]. With a single groove running from the center to the edge of the cell the tips split in all directions except that of the groove [10], in which a much faster growing dendritic structure is observed, and the whole pattern is very similar to that reported in Ref. [4]. An intrinsic anisotropy, such as that of a liquid crystal used as the more viscous fluid, has also been shown to stabilize the tips and yield growth with side branches $[11,12]$. All these experiments have demonstrated that different kinds of anisotropy affect viscous fingering as that of the surface tension does for dendritic crystal growth, i.e., stabilizing finger tips, so that, if the natural noise is strong enough, destabilization of the finger takes place only at its sides in the form of side branches.

In the channel geometry, Rabaud et al. have taken advantage of the fact that fingers remain stable up to higher capillary numbers once the introduced anisotropy has suppressed tip splitting to artificially induce side branching by means of an external perturbation [13]. This should enable one to study the side branching in a more controlled way, and also the coupling between the perturbation and the branching dynamics. They obtain side branches using a localized disturbance, namely, a knot on the thread that provides the anisotropy. Pressure modulation also causes side branching in the case of a thread, since, according to them, it mainly induces localized initial disturbances near the intersection of the interface with the thread. In contrast, in the case of two opposite grooves in the middle of the channel, the lateral waves caused by such sinusoidal pressure oscillations are symmetrical, and, most significantly, of limited amplitude.

This brings us to the fundamental problem of the general response of a pattern-forming interface to the nonlocalized periodic forcing of its dynamics. We study this response and the possible formation of lateral waves in an air finger invading a liquid crystal in the radial geometry, where the boundary conditions would not limit their amplitude, when periodically forcing the system by a modulated electric field 
perpendicular to the cell. A single groove running over the injection point stabilizes the finger tips in its direction. The nematic director tends to align with the electric field when this is switched on, and returns roughly to the cell plane when this is switched off. The flow properties depend on the orientation of the director, so that we expect to change the control parameters of the dynamics whenever we switch the field on or off. The use of a square wave for the amplitude of the electric field (switching it on and off instantly) enables us to observe the relaxation of the pattern to a parameter quench.

We find the tip radius to relax very quickly to two different values when the field is switched on and off, and that this pulsating tip induces symmetrical lateral undulations. Finally, we explain these lateral undulations as the trace of a periodic change in the selected tip radius, caused mainly by the change in the effective anisotropy due to the interplay between the liquid crystal and the groove. Back to the channel geometry, we confirm this scenario by numerically integrating a phase-field model for viscous fingering [14] in which the anisotropy is switched between two different values. Here, the alternate relaxation towards two different selected pattern scales is particularly clear, since the symmetrical lateral undulations saturate, as the finger oscillates between two different selected widths.

This mechanism might be relevant to experiments in which similar observations have been made. For instance, to the case of symmetrical undulations at the sides of a finger perturbed by a bubble on its tip, in which the tip curvature oscillates $[4,5,13]$ and the lateral undulations in the channel geometry also lie between two well-defined asymptotic widths, with a Saffman-Taylor finger as outer envelop [5,13]. Another example could be the sinusoidal modulation of the injection pressure in fingers grown with two parallel grooves, which also displayed symmetrical lateral waves of limited amplitude [13].

The rest of the paper is organized as follows: In Sec. II we present the experimental setup and observations. Section III then introduces and exploits the theoretical framework within which we explain these experimental results, and Sec. IV, the numerical method for checking the outcoming hypothesis in the channel geometry. The conclusions reached are summarized in Sec. V.

\section{EXPERIMENTAL SETUP AND RESULTS}

The experiments were performed in a radial Hele-Shaw cell. This was assembled from two glass plates coated with a conducting layer of $\mathrm{SnO}_{2}$, which served as electrode. The bottom plate, of dimensions $160 \mathrm{~mm} \times 160 \mathrm{~mm}$ and thickness $5.5 \mathrm{~mm}$, had a hole of $1 \mathrm{~mm}$ diameter in the center as an inlet for the air. On the coated face of the upper plate $(140 \mathrm{~mm} \times 140 \mathrm{~mm}$ and thickness $3.1 \mathrm{~mm})$ we engraved a groove following its diagonal. The plates were separated by $d=0.32 \mathrm{~mm}$ or $d=0.19 \mathrm{~mm}$ thick spacers. The inner faces of the plates corresponded to the coated ones, so that the electrodes directly faced each other, with no glass in between.

We applied an ac electric field $E$ of frequency $1 \mathrm{kHz}$ perpendicular to the plates, and switched it on/off with a frequency $\nu$. The "semiperiods" during which $E$ was on $\left(t_{o n}\right)$ and off were unequal. Their ratio was chosen so that fingers advanced a similar distance in each "semiperiod," which resulted in more apparent effects. Thus, a filling coefficient of $\xi=t_{\text {on }} \nu=0.67 \pm 0.03$ was found to be convenient, and it was used in all the experiments presented here.

Initially, the cell was filled with the commercial liquid crystal mixture RO-TN-430 (La Roche), with positive dielectric anisotropy, $\varepsilon_{a}=\varepsilon_{\|}-\varepsilon_{\perp}=17.6\left(\varepsilon_{\|}\right.$and $\varepsilon_{\perp}$ are the components of the uniaxial dielectric tensor parallel and perpendicular to the director, respectively), and a broad temperature range of the nematic phase, from $T_{m}=-10^{\circ} \mathrm{C}$ up to $T_{N \rightarrow I}=70^{\circ} \mathrm{C}$ (experiments were performed at room temperature $T=23^{\circ} \mathrm{C}$ ). The mixture was doped with dichroic blue dye $\mathrm{D} 16(\mathrm{BDH})$ in order to enhance the contrast at the air-nematic-liquid-crystal interface.

Then, after being filtered, air was injected through the hole of the bottom plate at an excess pressure $p_{e}$, regulated by a ported precision regulator (Norgren 11-818-100) with an accuracy of \pm 0.03 bar, and further decreased and stabilized by a unit for pressure reduction. The path of the air was regulated by two three-path solenoid valves, and $p_{e}$ was measured with a precision pressure meter (Watson \& Smith), with an accuracy of \pm 1 mbar.

As the air displaced the liquid crystal, a camera recorded the growth process, and images were fed into a PC for digital analysis, with a spatial resolution of 512 pixels $\times 512$ pixels and a 256 gray scale for each pixel. With the magnification used, a spatial resolution of $(0.241 \mathrm{~mm}$ $\times 0.166 \mathrm{~mm}) /$ pixel was determined.

Experimental results are presented in Figs. 1-4. In all cases, two air fingers whose tips do not split grow along the groove, at each side of the injection hole. Two much slower air bumps form at each side of the groove and perpendicular to it (Figs. 1, 3 and 4), and their tips can split [Fig. 4(a)].

At low excess pressures ( $p_{e}=5$ mbar, Figs. 1-3) the two stable viscous fingers along the groove do not show any lateral undulations with ( $E$ on) or without ( $E$ off) an ac field kept constant [Fig. 1(a) and Fig. 1(b), $d=320 \mu \mathrm{m}$, and Fig. 3(a) and Fig. 3(b), $d=190 \mu \mathrm{m}]$. However, fingers grown with the field [Fig. 1(b) and Fig. 3(b)] are thinner and slower (compare the times indicated in the captions) than their analogues grown without it [Fig. 1(a) and Fig. 3(a), respectively].

If one then periodically switches on and off the field (modulated $E$ ), the tips undergo successive curvature changes that induce formation of undulations at the sides of the two stable fingers in a strong correlation with the switching frequency $\nu$, as shown for two different ones in Fig. 1(c) and Fig. 1(d), where interfaces are displayed each time the field is switched on/off. Note that the maxima and minima of these lateral undulations in those figures roughly overlap with the profiles of the fingers grown in the same conditions but with the field kept off [Fig. 1(a)] and on [Fig. 1(b)], respectively.

Similar to the solidification of a nematic liquid crystal into a smectic $B$ reported in Ref. [15], above a certain switching frequency $\nu_{c}$ the main fingers show no lateral un- 

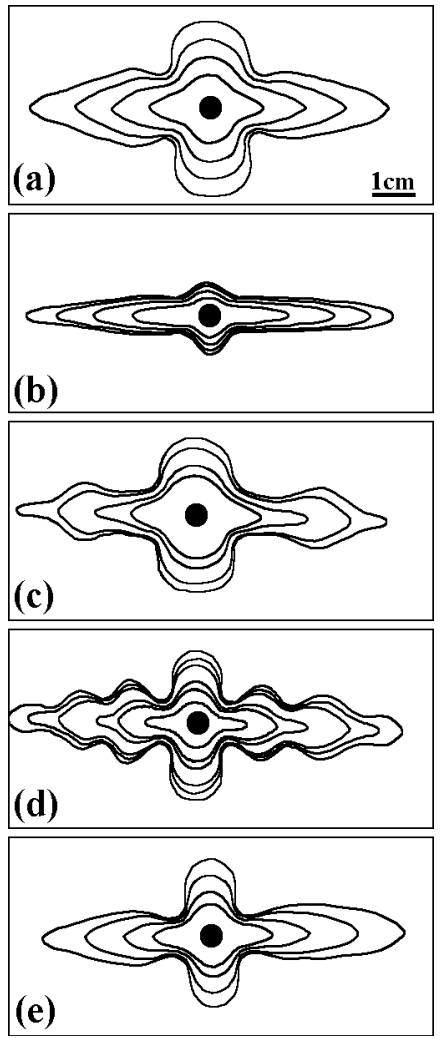

FIG. 1. Air-nematic interfaces at subsequent times. $d$ $=320 \mu \mathrm{m}, p_{e}=5$ mbar. (a) $E$ off, $t=0.32 \mathrm{~s}, 0.64 \mathrm{~s}, 0.96 \mathrm{~s}, 1.32 \mathrm{~s}$; (b) $E=0.32 \mathrm{~V} / \mu \mathrm{m}$ on, $t=1.08 \mathrm{~s}, 2.20 \mathrm{~s}, 3.32 \mathrm{~s}, 4.44 \mathrm{~s}$; (c) $-(\mathrm{e})$ modulated $E, \xi=0.68$ : (c) $\nu=0.667 \mathrm{~Hz}, t=0.4 \mathrm{~s}, 1.44 \mathrm{~s}, 1.84 \mathrm{~s}$, $2.64 \mathrm{~s}$; (d) $\nu=1.01 \mathrm{~Hz}, t=0.72 \mathrm{~s}, 1.04 \mathrm{~s}, 1.68 \mathrm{~s}, 2.04 \mathrm{~s}, 2.68 \mathrm{~s}, 2.88$ $\mathrm{s}$; (e) $\nu=4.55 \mathrm{~Hz}, t=0.52 \mathrm{~s}, 1.04 \mathrm{~s}, 1.56 \mathrm{~s}, 2.08 \mathrm{~s}$. (c) and (d) show the interfaces each time $E$ was switched on/off.

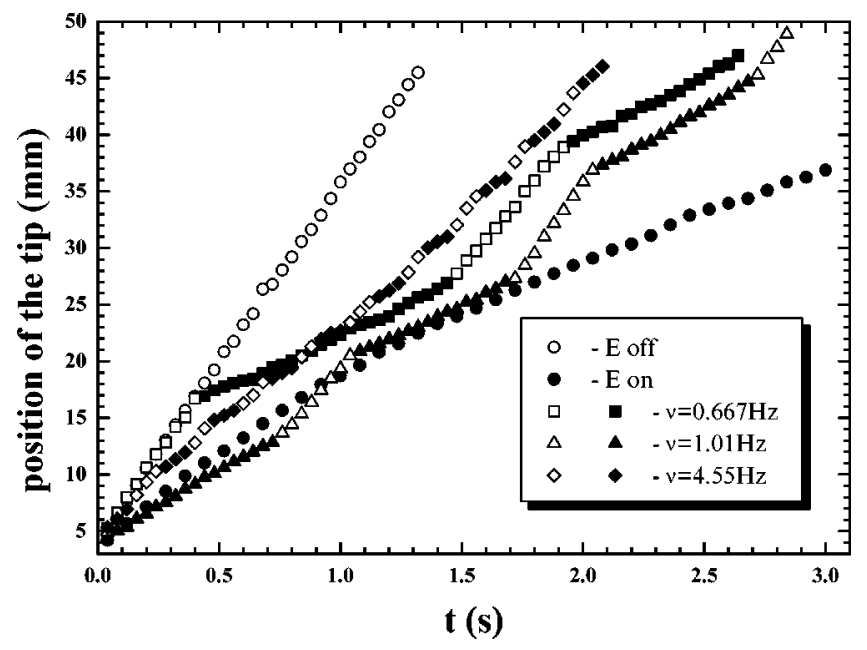

FIG. 2. Position of the tip of the main fingers in Fig. 1 vs time. Filled (empty) symbols denote $E$ on (off). Circles correspond to the experiments with field kept off (empty) of Fig. 1(a) or on (filled) of Fig. 1(b), whereas the other symbols stand for the different frequencies with which the field was switched on/off: squares, triangles, and diamonds for Figs. 1(c), 1(d), and 1(e), respectively.

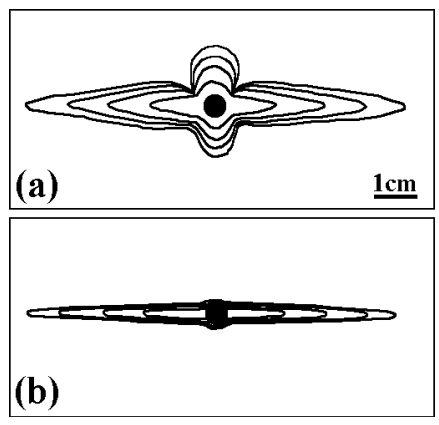

FIG. 3. Same as Fig. 1, but $d=190 \mu \mathrm{m}$. (a) $E$ off, $t=0.24 \mathrm{~s}$, $0.60 \mathrm{~s}, 1.00 \mathrm{~s}, 1.40 \mathrm{~s}$; (b) $E=0.55 \mathrm{~V} / \mu \mathrm{m}$ on, $t=0.60 \mathrm{~s}, 1.12 \mathrm{~s}, 1.72$ $\mathrm{s}, 2.24 \mathrm{~s}$.

dulations [Fig. 1(e)]. Their widths are then intermediate, lying between those of Fig. 1(a) ( $E$ off) and Fig. 1(b) ( $E$ on).

Figure 2 plots the position of the tip of the fingers in Fig. 1 vs time. Here it is apparent that fingers grow faster with $E$ off [wider fingers of Fig. 1(a), empty circles] than with $E$ on [thinner fingers of Fig. 1(b), filled circles], but also that the oscillations of the tip curvature in time when periodically switching on/off the field of Fig. 1(c) and Fig. 1(d) are accompanied by tip velocity oscillations (squares and triangles, respectively). In each oscillation, when the field is off (empty squares and triangles) and on (filled ones) the velocity roughly attains the values obtained for fingers grown with the field kept off (empty circles) and on (filled circles) all the time, respectively. Even for $\nu>\nu_{c}$, when no lateral undulations occur [Fig. 1(e)], the velocity increases and decreases significantly when the field is switched off (empty diamonds) and on (filled diamonds), respectively, although it is not clear whether it attains the same values than for a constant field.

With the same $p_{e}=5$ mbar but a smaller cell gap $d$ $=190 \mu \mathrm{m}$ (Fig. 3), all the previous qualitative observations are reproduced, but now all fingers are narrower [than in Fig. 1, compare Fig. 3(a) with Fig. 1(a) and Fig. 3(b) with Fig. $1(\mathrm{~b})]$.

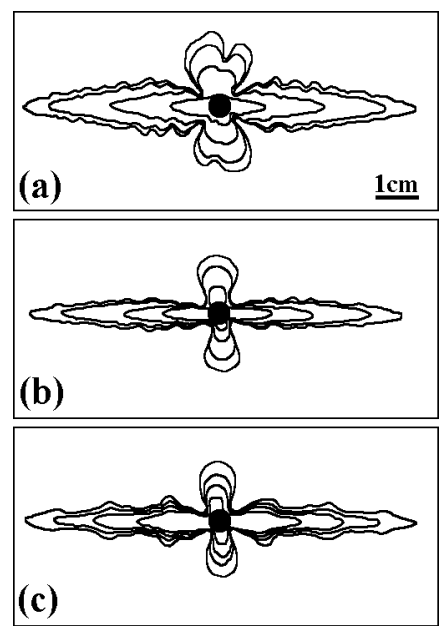

FIG. 4. Same as Fig. 3, but $p_{e}=22$ mbar. (a) $E$ off, $t=0.04 \mathrm{~s}$, $0.12 \mathrm{~s}, 0.20 \mathrm{~s}, 0.24 \mathrm{~s}$; (b) $E=0.55 \mathrm{~V} / \mu \mathrm{m}$ on, $t=0.08 \mathrm{~s}, 0.16 \mathrm{~s}, 0.28$ s, $0.36 \mathrm{~s}$; (c) modulated $E=0.58 \mathrm{~V} / \mu \mathrm{m}, \nu=8.42 \mathrm{~Hz}, \xi=0.68, t$ $=0.12 \mathrm{~s}, 0.20 \mathrm{~s}, 0.28 \mathrm{~s}, 0.36 \mathrm{~s}$. 
At higher excess pressure $\left(p_{e}=22 \mathrm{mbar}\right)$ and the same cell gap $d=190 \mu \mathrm{m}$ as in Fig. 3, the fingers along the grooves show a weak, uncorrelated lateral noise both with $E$ off and on [Fig. 4(a) and Fig. 4(b)], whereas their overall widths roughly stay the same [compare with Fig. 3(a) and Fig. 3(b), respectively]. When periodically switching the field on and off, the lateral undulations correlated with the switching frequency of Fig. 1(c) and Fig. 1(d) reappear, but now superimposed to and apparently decoupled from the uncorrelated lateral noise [Fig. 4(c)].

Also at this higher excess pressure $p_{e}=22 \mathrm{mbar}$ do the regular lateral undulations disappear above a certain frequency $\nu_{c}$ and leave the bare uncorrelated noise of Fig. 4(a) and Fig. 4(b). This upper frequency $\nu_{c}$ shows a roughly linear dependence on the excess pressure $p_{e}$.

\section{THEORETICAL DISCUSSION}

We now present a possible simplified theoretical framework to explain the experimental observations.

The shear viscosity of a nematic liquid crystal flowing in a planar cell depends on the orientation of its director: The highest viscosity is achieved with the director perpendicular to the cell (homeotropic alignment). With the director lying on the cell plane (planar alignment) the viscosity is lower and anisotropic: higher with the director perpendicular than parallel to the flow.

The director and the velocity fields are coupled by nonlinear nematohydrodynamic equations — see, e.g., Refs. $[16,17]$. Thus, when the electric field is off, the flow forces the director to be roughly in the plane of the cell (planar alignment case). Moreover, the director tends to align itself with the flow velocity in a certain pressure range. The latter, together with the mentioned anisotropy of the viscosity in the cell plane respect to the director orientation, results in a viscosity that depends on the velocity direction. This causes the viscosity to be nonuniform and anisotropic with respect to the direction of the flow. This anisotropy turns out to be the most important effect, since, if strong enough, it stabilizes the finger tips, thus switching from a tip splitting to a sidebranching mode [11]. This can be understood by mapping this anisotropy in the viscosity to an effective anisotropy in the surface tension [12].

However, experiments performed without any groove found no regime for which this anisotropy was strong enough to clearly stabilize the finger tips for the liquid crystal mixture used here [18], whereas the introduction of the groove did stabilize them. Therefore, as a first approximation, we will neglect the anisotropic effect of the director alignment in front of that of the groove: On one hand, we will consider this planar alignment case to have a unique, uniform, and isotropic average viscosity; on the other hand, we will not consider the effective anisotropy in the surface tension coming from that in the viscosity, but only the stronger anisotropy introduced by the groove. Actually considering both of them does not change qualitatively the simulation results.

An ac electric field also exerts a torque on the director. For a liquid crystal with positive dielectric anisotropy, $\varepsilon_{a}$
$>0$ as ours, and a field perpendicular to the plates, the electric torque competes with the shear one, trying to align the director with the field, i.e., perpendicular to the plates (homeotropic alignment). Therefore, the viscosity with $E$ on should now be even more isotropic in the shear plane.

Consequently, both with $E$ off and on we will consider a constant, isotropic viscosity. The only difference between the viscosities with and without electric field will be that the viscosity with $E$ on should always be larger than with $E$ off, since the field favors the homeotropic alignment. Note that this inequality of the viscosities with and without field will hold even for an incomplete alignment.

Thus, the theoretical framework will be that of the standard viscous fingering equations, except for the dimensionless surface tension $B_{0} \equiv \sigma /\left(p_{e} l_{c}\right)$ (with $\sigma$ the surface tension and $l_{c}$ an arbitrary length scale), which will read

$$
B=B_{0}\left(1-\alpha \cos ^{2} \phi\right),
$$

where $\phi$ is the angle between the single groove used in the experiments and the normal to the interface, and $\alpha$ represents the twofold anisotropy induced by the groove. Grooves and grids have usually been modeled by such an anisotropy in the surface tension (see, e.g., Ref. [19]). This represents a strong simplification, but we do not expect it to affect the conclusions of this paper in any fundamental way.

Each time we switch $E$ on or off, we change the director orientation, and thus some physical parameters of the model, which should result in a change in its dimensionless control parameters, namely, $B_{0}$ and $\alpha$, or in the time scale of the dynamics, $12 \mu l_{c}^{2} /\left(d^{2} p_{e}\right)$, where $\mu$ is the viscosity. Since we always switch $E$ on or off instantly, the adimensionalization leading to this set of control parameters remains valid, even if their value is periodically switched. Before using evidence from the experiments, let us discuss for clarity how can these control parameters and time scale be expected to change $a$ priori.

The time scale $12 \mu l_{c}^{2} /\left(d^{2} p_{e}\right)$ can change only through a change in the viscosity $\mu$. Indeed, the change in the time scale was measured directly for the same mixture from the growth of a circular interface in the absence of grooves with $E$ on and off, and it was found to be a factor 3.7 slower with $E$ on [18]. We thus know that the viscosity is 3.7 times larger with $E$ on. (Note that this does not alter $B_{0}=\sigma /\left(p_{e} l_{c}\right)$, since it does not depend on $\mu$ for an experiment at constant excess pressure, whereas it does for the constant injection rate case, for which its definition is different). $B_{0}=\sigma /\left(p_{e} l_{c}\right)$ could only be altered by a variation in the surface tension $\sigma$. Such a variation has been measured for several liquid crystals, and $\sigma$ has been found to be $20-50 \%$ smaller with the director parallel to the air-nematic interface (roughly our $E$ on case) than perpendicular to it [20] (closer to $E$ off).

As for the anisotropy $\alpha$ due to the groove, it could be changed by the following effect: The director inside the groove might keep the planar alignment to some extent even with $E$ on, since the conducting layer was removed from the etched region when engraving the groove. In that case, the viscosity would be lower inside than outside the groove with $E$ on, thus reinforcing the mobility enhancement of the 
groove itself (higher gap $d$ ), and, therefore, increasing the effect of the groove (the anisotropy $\alpha$ in our model).

Now, consider the experimental results reported in the previous section, and first of all, those for a field kept either on or off during all the experiment. The main fingers were found to be slower and thinner with $E$ on. Their smaller velocity is explained by the increase in the time scale of the dynamics due to that in the viscosity, whereas their smaller width should be understood as a decrease in the selected finger tip radius for a given length of the finger, which results in a visually overall thinner finger. (Thus we will talk about thinner and wider fingers to refer to larger and smaller tip curvatures at a given finger length, respectively). In our model, this decrease in the selected length scale could be due to either a decrease in the dimensionless surface tension $B_{0}$ or an increase in its anisotropy $\alpha$.

To check the two possibilities, we varied $B_{0}$ and $\alpha$ by means other than reorienting the director. In order to increase $\alpha$, we decreased the cell gap $d$, which is the standard way of increasing the effect of a groove or grid [6,19], and which does not affect anything else in our model but the time scale. As mentioned in the previous section, the fingers do narrow. Note also that very similar widths are obtained either by switching on the field [Fig. 1(b)] or by increasing $\alpha$ through a decrease in $d$ [Fig. 3(a)]. This proves that the observed finger narrowing when switching on the field can be caused by an increase in $\alpha$. Consistently with this hypothesis, if $\alpha$ is further increased by switching on the field with this lower cell gap $d$, the fingers narrow more [Fig. 2(b)].

In order to decrease $B_{0}$, we kept this lower cell gap and increased the injection pressure up to $p_{e}=22$ mbar. $B_{0}$ must have been actually lowered, since the interfaces obtained were much noisier, and the fingers growing perpendicular to the groove even tip split, as reported in the previous section. (The amount of noise necessary for a finger to tip split is known to decrease with decreasing dimensionless surface tension $B_{0}$ [21]). However, also as explained in the previous section, there was no significant width change. The fact that a change by a factor 4.4 in the dimensionless surface tension $B_{0}$ when increasing the injection pressure from $p_{e}=5 \mathrm{mbar}$ up to $p_{e}=22$ mbar causes no visible width change implies that the mentioned change of $20-50 \%$ in $B_{0}$ through the change in the surface tension $\sigma$ measured for other liquid crystals cannot cause it either. We are, therefore, led to conclude that it is the anisotropy in the surface tension and not the dimensionless surface tension itself what accounts for the observed width change.

Once we have understood how the introduction of an electric field affects the width and velocity of the fingers, we are in a position to explain the experimental observations when the electric field is periodically switched on and off. One would be tempted to understand the lateral oscillations in Fig. 1(c), Fig. 1(d), and Fig. 4(c) as standard side branches, i.e., due to the amplification of perturbations originating on the tip of the fingers. One could thus think that the periodic change in some control parameter when switching on and off the field provided the necessary local perturbation on the tips to induce side branching, or that a large enough perturbation due to the natural noise was further amplified through a resonance phenomenon with the frequency of change of this parameter and thus also produced visible and regular side branches, as seen in related problems [15]. In this case, the relevant control parameter should be the anisotropy $\alpha$, since the viscosity only enters the time scale, and can thus not affect the shape of the pattern. In this scenario, the fact that Fig. 1(e) shows no oscillations would be interpreted as the result of being too far from the resonance frequency, and the velocity oscillations seen in Fig. 2 would be those sometimes associated with side branching.

However, the velocity turns out to decrease when the finger tip narrows, as opposite to the usual case. This smaller velocity of thinner tips can only be explained by the change in the time scale due to the change in the viscosity. Actually, we visually observe the velocity to change instantly each time the field is switched on and off in each period, and it roughly attains in each semiperiod when the field is on (off) the same value than in a finger grown all the time with $E$ on (off), as explained in the previous section.

The finger tips are also observed to narrow at the very moment the field is switched on and to widen at the moment it is switched off, and the minima (maxima) of the lateral undulations also have approximately the same width than the finger with $E$ kept on (off), as also explained above.

All this suggests that the lateral undulations are the wake left by a tip quickly and alternately relaxing to the two different selected radii corresponding to the two different values (one for $E$ on and one for $E$ off) of the relevant control parameter, the groove anisotropy $\alpha$.

Thus, the obtained undulated fingers when periodically switching on and off the field of Fig. 1(c) and Fig. 1(d) themselves are the result of alternately relaxing between the thinner [Fig. 1(b)] and wider [Fig. 1(a)] fingers grown all the time with or without the field, respectively. With this explanation, the absence of significant lateral undulations for too high frequencies is due to the lack of time for the finger to relax to any of the two widths within each period, which should result in an intermediate width, as is indeed the case in Fig. 1(e).

This mechanism seems to be decoupled from "natural" (noise induced) side branching, since, when this natural side branching is already present with a field kept off and on [Fig. 4(a) and Fig. 4(b), respectively], periodically switching on and off the field seems just to superimpose the mentioned wake of tip radius changes, but not to eliminate or regularize the previously present modes [Fig. 4(c)]. The fact that the two effects be decoupled supports the idea that the width and velocity oscillations observed when switching on and off the field are the result of the relaxation back and forth between two different stationary widths and velocities, rather than that of the amplification of perturbations coming from the tip.

Indeed, instantly switching on and off the field with a certain period does not introduce any extra time scale nor control parameter in the dynamics of each semiperiod during which the field is either on or off. Each semiperiod can be understood as the relaxation with certain values of the control parameters towards a new steady state from a given initial condition. Just that this initial condition turns out to be a 
state more or less close to the steady state corresponding to different values of the control parameters. Since no extra time scale is introduced, no coupling with the natural noise was to be expected.

However, varying the amplitude of the field with say a sinusoidal wave instead of a square one would introduce as new time scale the period of the wave, so that the dynamics would change. The injection pressure in viscous fingering in a channel has indeed been varied with such a sinusoidal wave. The interesting point is that the tip velocity follows the pressure modulation and the pattern obtained also displays lateral undulations of limited amplitude, which are symmetrical as long as no external element breaks this symmetry (case of two parallel grooves) [13]. Also a bubble on the tip of a finger can induce the tip curvature to oscillate periodically and give rise to symmetrical lateral undulations of a well-defined amplitude (in the channel geometry) and periodicity. The amplitude is such that the outer envelope of the wave is a larger Saffman-Taylor finger in the channel, and the periodicity is correlated to the frequency of oscillation of the tip $[4,5,13]$.

All these observations with pressure modulation or bubbles match our own observations with electric field modulation, so that the mechanism of successive relaxations between two different steady states that we propose might also be relevant to these other experiments. Our case, however, is particularly clear thanks to the use of a square wave to modulate the electric field.

In conclusion, two different limiting cases seem to lead to the formation of lateral waves: (i) The amplification of small perturbations when advected from the tip to the sides of the finger (e.g., natural, noise-induced side branching). (ii) Successive and alternate relaxations between two different finger widths, also advected from the tip to the sides, when for some reason the tip curvature oscillates (e.g., periodic, instant changes in a control parameter affecting selection as in our experiments). Of course, the lateral undulations caused by the successive relaxations (ii) might also be damped or amplified as in (i), and it can be difficult to tell whether a particular deformation of the tip is rather a small perturbation (i) or an overall curvature change (ii), so that we feel that both mechanisms should be regarded as complementary, and experiments where a large perturbation is used to force the dynamics might be expected to be mixed cases.

\section{NUMERICAL RESULTS AND DISCUSSION}

The difficulty to check the explanation of the lateral undulations in terms of successive changes in the selected width proposed in the previous section lies in the fact that the finger width is not well defined. The sides of (anisotropic) viscous fingers in the radial geometry are not parallel, and, most importantly, anisotropic fingers do not reach a steady tip radius nor velocity. There is indeed a selection mechanism, but the first keeps growing and the latter decreasing with time (see Ref. [22]). Therefore, it is especially useful to perform numerical simulations of anisotropic fingers in the channel geometry to check out this scenario, since their sides are parallel, and, above all, their widths and velocities do saturate and are easy to compare with one another. Note that the experiments in the channel geometry with pressure modulation or bubbles mentioned in the previous section [13] are not clear enough for that purpose, since the selected width keeps changing all the time as the effective control parameters should oscillate sinusoidally in response to a sinusoidal pressure or bubble forcing. In contrast, we will instantly change the value of the relevant control parameter (the anisotropy due to the groove $\alpha$ ) in our simulations to mimic the switching on and off of the electric field.

On the other hand, it is well known that a thinner finger grows faster in dimensionless time, although the experimental observation is just the opposite in real time. This means that the change in the time scale due to the change in the viscosity (3.7 times larger with $E$ on) must be dominant over the change in dimensionless time. The question is whether there actually exists a range of change of the groove anisotropy $\alpha$ that yields the observed narrowing of the finger but also respects the fact that thinner fingers grow slower in real time.

To answer this question and check the proposed explanation of the lateral undulations, we numerically integrate the described theoretical model, but we run it in the channel geometry. We use the phase-field model for viscous fingering presented and tested in Ref. [14]. The only change in the model is that we now use the anisotropic surface tension given by Eq. (3.1). We recall the model,

$$
\begin{gathered}
\tilde{\epsilon} \frac{\partial \psi}{\partial t}=\nabla^{2} \psi+c \vec{\nabla} \cdot(\theta \vec{\nabla} \psi)+\frac{1}{\epsilon} \frac{1}{2 \sqrt{2}} \gamma(\theta)\left(1-\theta^{2}\right), \\
\epsilon^{2} \frac{\partial \theta}{\partial t}=f(\theta)+\epsilon^{2} \nabla^{2} \theta+\epsilon^{2} \kappa(\theta)|\vec{\nabla} \theta|+\epsilon^{2} \hat{z} \cdot(\vec{\nabla} \psi \times \vec{\nabla} \theta),
\end{gathered}
$$

where $\psi$ is the stream function, $\theta$ is the phase field, $c \equiv(\mu$ $\left.-\mu_{0}\right) /\left(\mu+\mu_{0}\right)$ is the viscosity contrast $\left(\mu, \mu_{0}\right.$ are the viscosities of the liquid crystal and the air, respectively) and $\epsilon$, $\tilde{\epsilon}$ are model parameters that must be small to recover the sharp-interface equations of the theoretical model. We have defined $f(\theta) \equiv \theta\left(1-\theta^{2}\right)$, and $\gamma(\theta) / 2 \equiv \hat{s}(\theta) \cdot\{\vec{\nabla}[B(\theta) \kappa(\theta)]$ $+\hat{y}\}, \kappa(\theta) \equiv-\vec{\nabla} \cdot \hat{r}(\theta)$, with $B(\theta) \equiv B[\phi=\arccos \hat{y} \cdot \hat{r}(\theta)]$, $\hat{r}(\theta) \equiv \vec{\nabla} \theta /|\vec{\nabla} \theta|$ and $\hat{s}(\theta) \equiv \hat{r}(\theta) \times \hat{z}$. All quantities are dimensionless and, in particular, lengths are in units of the channel width ( $y$ is length along the channel, $x$ across it, $\hat{z}$ is perpendicular to the plates, and $\phi$ is reinterpreted as the angle between $\hat{y}$ and the normal to the interface).

We set $B_{0}=10^{-2}$, which we know to allow stable fingers for the amount of numerical noise present even for vanishing anisotropy [12]. We use two different values of the anisotropy, $\alpha=0.9$ and $\alpha=0.1$, to account for the cases with and without electric field, respectively. The higher anisotropy gives the lowest $B$ at the finger tip that we will need to resolve, and thus the value of the interface width to use, $\epsilon$ $=0.00625$. As for the viscosity contrast, for numerical convenience we use $c=0.9$, which is known (see, e.g., Ref. [12]) 

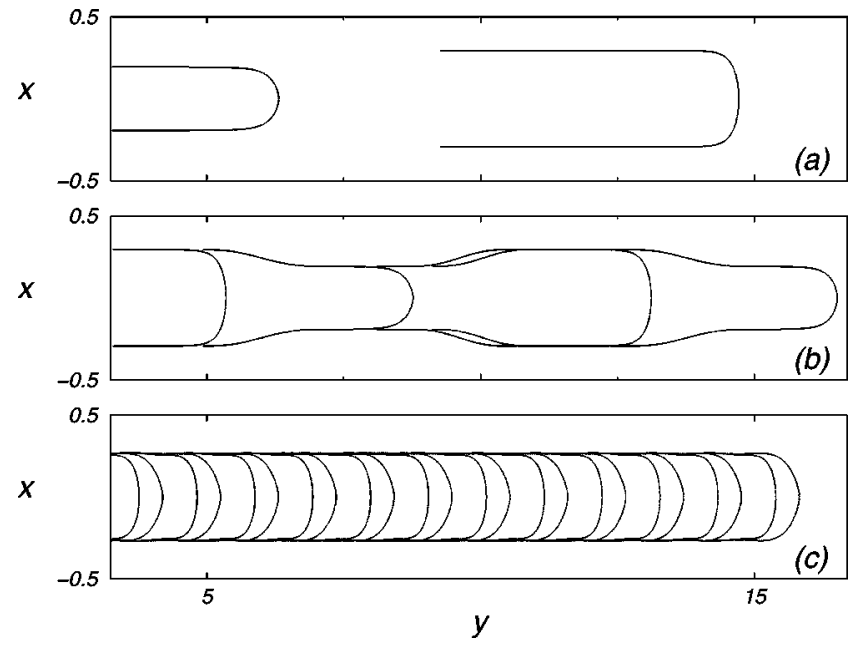

FIG. 5. Interfaces in the channel geometry, simulated in the reference frame moving with the mean interface and then translated into the laboratory frame. $B_{0}=10^{-2}, \epsilon=0.00625, c=0.9, \tilde{\epsilon}=0.4$. (a) Change of width in the stationary pattern at $t^{\prime}=7.8$ when changing from $\alpha=0.1$ (wider finger) to $\alpha=0.9$ (thinner finger). (b),(c) Periodic, instantaneous switch of $\alpha$ between the two values in (a), with a lower (b), and a higher (c) frequency. Interfaces are shown each time the field is switched on or off with $\xi=0.67$, until $t^{\prime}$ $=15.3$.

to be sufficiently close to the high viscosity contrast limit $c$ $=1$ of the experiments. $\tilde{\epsilon}=0.4$, which suffices to resolve the displacement of the liquid crystal by the air. The initial condition is a cosine wave of wavelength and amplitude 1 (the channel width) in all cases.

Since the simulations use dimensionless variables, the effect of the different time scale with or without the electric field does not show up. To make it apparent, we introduce another dimensionless time increment

$$
\Delta t^{\prime} \equiv \begin{cases}\Delta t & \text { when field is off } \\ a \Delta t & \text { when field is on }\end{cases}
$$

where $a=3.7$ is the measured ratio of the time scale with $E$ on and that with $E$ off, and we compare runs at a same time $t^{\prime}$. In this way we compare runs that would have taken the same time in the experiments, since the factor restoring the dimensions is now the same independently of how much time was the field on or off during each run. Also, the phasefield equations [Eqs. (4.1) and (4.2)] are in the reference frame moving with the mean interface. Since the experimental figures are in the lab frame, the numerical simulations (Figs. 5-7) have been translated into the latter for comparison.

Figures 5 and 7 are the computational, channel analogues of Fig. 1 and Fig. 2, obtained from experiments in the radial geometry. In Fig. 5(a) we show a wider ( $\alpha=0.1$, field off, $\lambda=0.588$ ) and a thinner ( $\alpha=0.9$, field on, $\lambda=0.387$ ) finger, both at $t^{\prime}=7.8$, where $\lambda$ is the finger width. We can see that the wider finger does go faster in real time even for this significant change in width. Therefore, we conclude that a

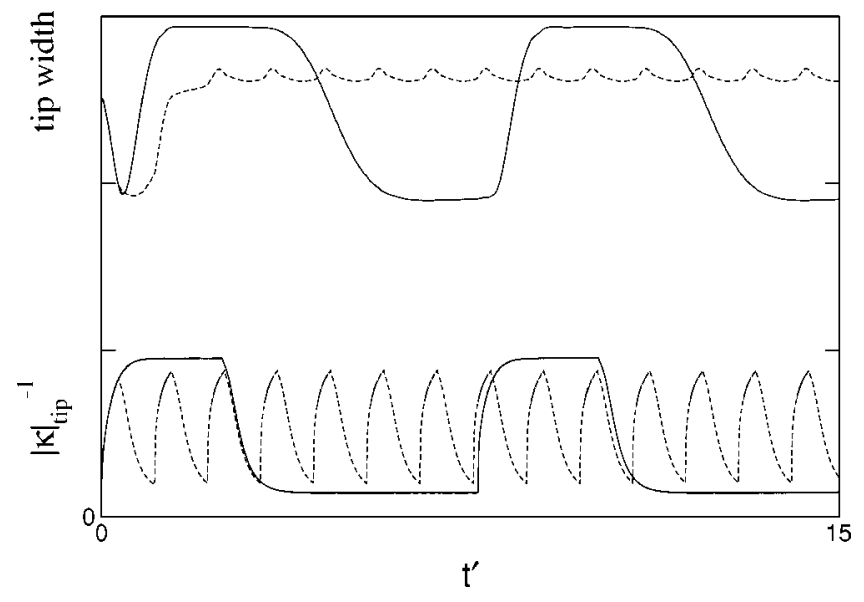

FIG. 6. Finger width at one unit length behind the tip and tip radius vs rescaled time $\left(t^{\prime}\right)$ for the runs in Fig. 5(b) (solid lines) and Fig. 5(c) (dashed lines). Recall that the unit length is the channel width.

simultaneous increase in the surface tension anisotropy and the viscosity, does actually explain the fact that fingers are both narrower and slower.

In Fig. 5(b) and Fig. 5(c), interfaces are shown exactly each time the anisotropy was changed between the two different values in Fig. 5(a) (each time the field was switched on or off), which was done with a very similar filling coefficient than in the experiments, $\xi=0.67$. This visually leaves no doubt of the fact that the two different widths in Fig. 5(a) are successively selected at the tip to produce the pattern in Fig. 5(b). The small mismatch between the tails of the two front interfaces in Fig. 5(b) is presumably due to the fact that the viscosity contrast is not strictly $1(c=0.9)$, so that the dynamics in the tail region is not completely frozen. In Fig. 5(c) the width has no time to relax to any of the two in Fig. $5(\mathrm{a})$, and gently oscillates in the intermediate range 0.526 $<\lambda<0.537$. However, the curvature seems to relax more quickly.

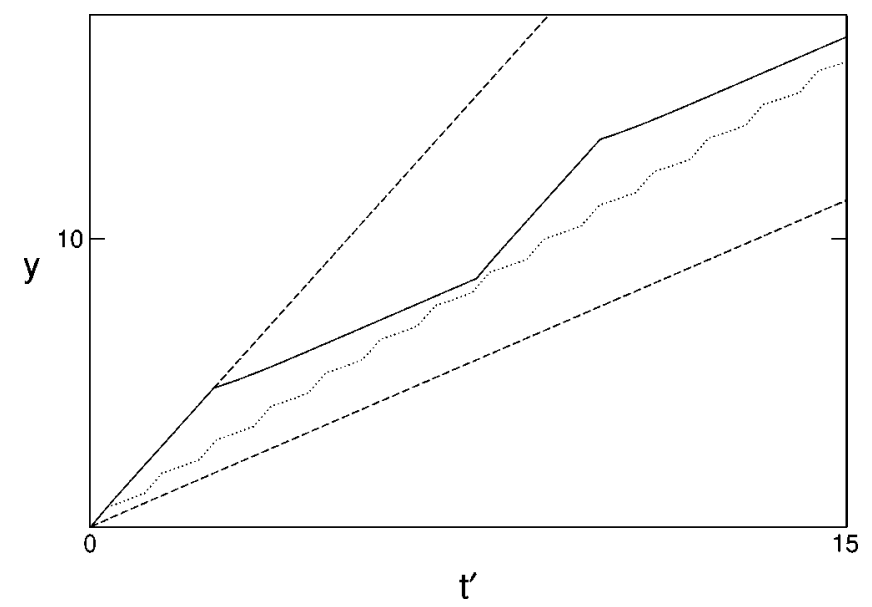

FIG. 7. Tip position $(y)$ vs rescaled time $\left(t^{\prime}\right)$ for the runs in Fig. 5. The steeper (less steep) straight, long-dashed line corresponds to the wider (thinner) finger in Fig. 5(a). The solid and dotted lines in between correspond to the runs in Figs. 5(b) and 5(c), respectively. 
These relaxation processes can be seen in more detail in Fig. 6, where we have monitored the finger width one unit length behind the tip (which is only slightly below the asymptotic width) and the tip radius. The latter is the inverse of the curvature modulus of the zero level-set of the phase field $[1 /|\kappa(\theta=0)|]$, and, therefore, of the interface. To avoid spurious lattice oscillations, such a radius is plotted only when the finger tip hits near a grid point $\left(|\theta|<10^{-3}\right.$ at the tip of the finger). The solid and dashed lines correspond to the runs in Fig. 5(b) and Fig. 5(c), respectively. In the case of the lower frequency (solid lines), we can see that the tip radius relaxes always first to its asymptotic value, and is then followed by the finger width as the information of the tip is left behind. Thus, for the higher frequency (dashed lines), the finger has not enough time to relax to its asymptotic widths, but the curvature almost attains its asymptotic values. On the other hand, for the lower frequency it is possible to observe that the tip widens much faster than it narrows, as can be seen both in the tip width and its radius, but especially in the first. This behavior may be expected in connection with the existence of a set of (unstable) solutions with larger width than the selected one, whose proximity may effectively slow down the relaxation dynamics.

Finally, in Fig. 7 we show the evolution of the tip position for the runs in Fig. 5. The steeper (less steep) straight, longdashed line corresponds to the wider (thinner) finger in Fig. 5(a), i.e., to the case with the field off (on). The initial relaxation to the stationary velocity is so fast that it is almost invisible at this scale. The runs in Figs. 5(b) and (c) correspond to the solid and dotted lines in between, respectively. We can see that, for the lower frequency (solid line), the velocity successively relaxes to the values with or without field of the straight, long-dashed lines. Initially, however, it attains a value slightly below (above) the steady velocity when it relaxes to a lower (higher) velocity. This effect is more apparent for the relaxation to a lower velocity. In contrast, for the higher frequency (dotted line), we are left with these slightly too low or high initial values of the velocity, since the field is switched on or off again just when the velocity was about to achieve its asymptotic value. This is quite similar to what happened to the curvature for the higher frequency (lower dashed line in Fig. 6), in contrast with the failure of the finger width to relax (upper dashed line in Fig. 6 ). So the finger velocity seems to be more correlated to the tip curvature than to the finger width.

In order to compare with the experiments of Rabaud et al. with two opposite grooves in the channel geometry in which they modulated the injection pressure [13], we have repeated our simulations changing the dimensionless surface tension from $B_{0}=10^{-2}$ to $B_{0}=6.5 \times 10^{-4}$ (with $\epsilon=0.005$ ) and keeping its anisotropy to $\alpha=1$. Note that, indeed, an instant change in pressure is equivalent to a change in the time scale and the dimensionless surface tension. In the experiments of Rabaud et al. the modulation was sinusoidal, which also introduces an extra time scale, but they nevertheless obtained symmetrical lateral waves of limited amplitude as ours [13]. In our simulations, we use instant changes in the dimensionless surface tension, and we obtain qualitatively the same results than in Fig. 5 and Fig. 7. The instant changes make the saturation of the finger width possible, and the fact that the finger width does saturate to the values with a constant $B_{0}$ suggests that the basic mechanism for the lateral waves observed by Rabaud et al. when modulating the pressure might also be the relaxation towards two different steady widths.

\section{CONCLUSIONS}

We have performed viscous fingering experiments in a radial Hele-Shaw cell, where the more viscous fluid was a liquid crystal mixture in its nematic phase. After ruling a single groove across the center of the cell, we achieved stable finger tips in the direction of the groove (otherwise unstable). By applying an electric field perpendicular to the cell, we oriented the nematic director in this direction, which resulted in thinner and slower fingers. We then periodically switched on and off the field to find oscillations in the finger width and velocity, with an amplitude that decreased as the switching frequency was increased.

We explain how fingers are slower when the field is on because the viscosity of the liquid crystal is higher with the director perpendicular to the cell, and that the reason why they are thinner may be attributed to an increase in the anisotropy due to the groove when the field is on. Also the surface tension is reduced when the field is switched on, but it cannot affect so strongly the finger width, since no significant width change was observed by increasing the excess pressure, and both a decrease in the surface tension and an increase in the excess pressure would lower the dimensionless surface tension. The proposed scenario reproduces the experimental observations, as shown by numerical integration in the channel geometry of a simplified theoretical model. We also explain the finger width and velocity oscillations as the result of the relaxation back and forth between the selected tip radii and velocities with the field on and off, as suggested by the experiments and clearly seen in the numerical integration of the theoretical model.

We discuss how this latter result might be relevant to experiments with a bubble on the tip of a finger and especially when modulating the injection pressure in a channel with two parallel grooves $[4,5,13]$. We reproduce the qualitative observation that the lateral waves are symmetric and of limited amplitude for such a pressure modulation by simulations instantly changing the dimensionless surface tension. We point out that the amplification of small tip perturbations describing natural, noise-induced side branching, and the successive relaxations between to steady widths describing the formation of lateral undulations when periodically changing a control parameter seem to be two complementary mechanisms for lateral wave formation, and that experiments forcing the dynamics with large perturbations might be understood as mixed cases.

\section{ACKNOWLEDGMENTS}

We acknowledge financial support from the Dirección General de Enseñanza Superior (Spain), Project Nos. 
BXX2000-0638-C02-02 and BFM2000-0628-C03-01, from the National Scientific Research Foundation (Hungary), Grant Nos. OTKA F022771 and OTKA T031808, and the European Commission, Project No. ERB FMRX-CT96-
0085. Simulations have been carried out using the resources at CESCA and CEPBA, coordinated by $\mathrm{C}^{4}$. R.F. also acknowledges a grant from the Comissionat per a Universitats i Recerca (Generalitat de Catalunya).
[1] E. Ben-Jacob and H. Levine, Adv. Phys. 49, 395 (2000); J. P. Gollub and J. S. Langer, Rev. Mod. Phys. 71, S396 (1999); Solids far From Equilibrium, edited by C. Godrèche (Cambridge University Press, Cambridge, 1992); D. A. Kessler, J. Koplik, and H. Levine, Adv. Phys. 35, 255 (1988); P. Pelcé, Dynamics of Curved Fronts (Academic, New York, 1988).

[2] K. McCloud and J. Maher, Phys. Rep. 260, 139 (1995).

[3] L. Paterson, J. Fluid Mech. 113, 513 (1981).

[4] Y. Couder, O. Cardoso, D. Dupuy, P. Tavernier, and W. Thom, Europhys. Lett. 2, 437 (1986).

[5] Y. Couder, N. Gerard, and M. Rabaud, Phys. Rev. A 34, 5175 (1986).

[6] E. Ben-Jacob, R. Godbey, N. Goldenfeld, J. Koplik, H. Levine, T. Müller, and L. Sander, Phys. Rev. Lett. 55, 1315 (1985).

[7] E. Ben-Jacob and P. Garik, Nature (London) 343, 523 (1990).

[8] J. Chen and D. Wilkinson, Phys. Rev. Lett. 55, 1892 (1985); J. Chen, Exp. Fluids 5, 363 (1987).

[9] V. Horváth, T. Vicsek, and J. Kertész, Phys. Rev. A 35, 2353 (1987).

[10] M. Matsushita and H. Yamada, J. Cryst. Growth 99, 161 (1990).

[11] See, e.g., Á. Buka, in Pattern Formation in Liquid Crystals, edited by Á. Buka and L. Kramer (Springer, New York, 1996), p. 291.
[12] R. Folch, J. Casademunt, and A. Hernández-Machado, Phys. Rev. E 61, 6632 (2000).

[13] M. Rabaud, Y. Couder, and N. Gerard, Phys. Rev. A 37, 935 (1988).

[14] R. Folch, J. Casademunt, A. Hernández-Machado, and L. Ramírez-Piscina, Phys. Rev. E 60, 1724 (1999); ibid. 60, 1734 (1999).

[15] T. Börzsönyi, T. Tóth-Katona, Á. Buka, and L. Gránásy, Phys. Rev. Lett. 83, 2853 (1999).

[16] H. Pleiner and H. Brand, in Pattern Formation in Liquid Crystals, edited by Á. Buka and L. Kramer (Springer, New York, 1996), p. 15.

[17] E. Dubois-Violette and P. Manneville, in Pattern Formation in Liquid Crystals (Ref. [16]), p. 91.

[18] T. Tóth-Katona and Á. Buka (unpublished).

[19] E. Ben-Jacob, P. Garik, T. Mueller, and D. Grier, Phys. Rev. A 38, 1370 (1988); E. Ben-Jacob and P. Garik, Physica D 38, 16 (1989).

[20] V. A. Tsvetkov, O. V. Tsvetkov, and V. A. Balandin, Mol. Cryst. Liq. Cryst. Sci. Technol., Sect. A 329, 305 (1999).

[21] D. Bensimon, Phys. Rev. A 33, 1302 (1986).

[22] R. Almgren, W.-S. Dai, and V. Hakim, Phys. Rev. Lett. 71, 3461 (1993). 